\title{
A Novel Decoupling Control of Muti-Axis Shaking Table based on the Modified Adaptive Notch Filter
}

\author{
Zhang Yanyan ${ }^{1, a}$, Yang Zhidong ${ }^{2, b}$ * \\ ${ }^{1}$ Harbin Institute of Technology, Harbin, China \\ ${ }^{2}$ Harbin Institute of Technology, Harbin, China \\ azhangyanyan963@163.com, b yangzhidong@hit.edu.cn
}

Keywords: MAST; earthquake waveform; dynamic cross coupling; active noise control; adaptive notch filter

Abstract. The multi-axis shaking table (MAST) is widely used in the vibration tests for high-rise buildings, bridges, large structures and so on. Due to the overturning moment and off-center load of the MAST, the dynamics cross-coupling disturbance occurs when the motion on one translational degree of freedom (DOF) couples into another rotary DOF or vice versa. The cross-coupling will reduce the tracking accuracy of the command waveform. A novel decoupling control for the sinusoidal command waveform and sweep sine command waveform is proposed based on the modified adaptive notch filter. The approach, inspired by the active noise control technology, takes the coupling motion as the noise for the coupled DOF, and cancels the cross-coupling disturbance by adding harmonics of the same frequency to the coupling motion, along with just the right phase and amplitude. A model of the plant is introduced to give the method critical phase information needed to adapt canceller coefficients. The experimental results indicate that the decoupling control can effectively reduce the dynamics coupling between the translational and rotary DOFs of the MAST.

\section{Introduction}

The shaking table is a valid equipment for the structure fatigue test and earthquake simulation ${ }^{[1,2]}$. It can be used for analyzing the damage mechanism of structures under earthquake waveform. Due to the overturning moment and off-center load of the MAST, the dynamics cross-coupling disturbance occurs when the motion on one translational degree of freedom (DOF) couples into another rotary DOF or vice versa. Wei ${ }^{[3]}$ and Yang ${ }^{[4]}$ have established the linear model of the hydraulic servo system and analyzed it. The coupling force observation based on the coupling model is introduced into the DoFs control structure. The coupling forces are taken as disturbance forces distributed into each actuator of the hydraulic system through the Jacobian matrix transformation. And the decoupling control is based on the dynamics model as well as a feedforward disturbance force compensation control strategy ${ }^{[5]}$. Moreover, due to the fact that differentiating acceleration which contains large noise is needed for the decoupling control based on the dynamics model, the modal decoupling control is given. Modal equations of the redundant shaking table are given by considering the hydraulic cylinder as a hydraulic spring. Through the standard modal matrix and its inverse matrix, the redundant shaking table can be controlled in the non-coupling modal space instead of DoFs space $^{[6]}$. Besides Tagawa ${ }^{[7]}$ has reduced the cross coupling disturbance, which iterates the disturbance out by playing the command waveform repeatedly, observing the response, and updating the system drive waveform using the system inverse frequency response function. This method is quite effective, but can be very time consuming and requires a significant level of user interaction and sophistication. This paper will focus on cancelling the cross-coupling disturbance for the sinusoidal command waveform and swept sine command waveform, and a novel decoupling control method is proposed based on the modified adaptive notch filter. The control method takes the coupling sinusoidal motion as the noise for the coupled DOF, and a modified adaptive notch filter is designed to eliminate the motion disturbance by adding harmonics of the same frequency to the coupling motion, along with just the right phase and amplitude. A model of the plant is introduced to give the method critical phase information needed to adapt canceller coefficients. 


\section{Basic Principle of Adaptive Notch Filter}

The adaptive notch filter is originated from the adaptive noise cancellation, which adds the sinusoidal harmonics with just the right phase and amplitude to the primary signal including a noise signal to cancel the noise component ${ }^{[8,9,10]}$. And a optimized notch filter for sinusoidal noise is derived $^{[11]}$.

The adaptive notch filter noise canceller, which utilizes the Least Mean Square (LMS) recursive algorithm, is shown in Fig.1. The concept is based on the availability of two separate signal sources, that is the primary signal and reference signal. The primary signal is obtained from the sensor which receives both the desired signal and the noise signal. The reference signal is a noise input (i.e. a sinusoid) which is uncorrelated with the desired signal but somehow correlated with the noise component received by the primary sensor. And the adaptive filtering process produces an output that is the same as the noise component in the primary input. Thus when the output of the adaptive filter is subtracted form the primary signal, the noise component is eliminated.

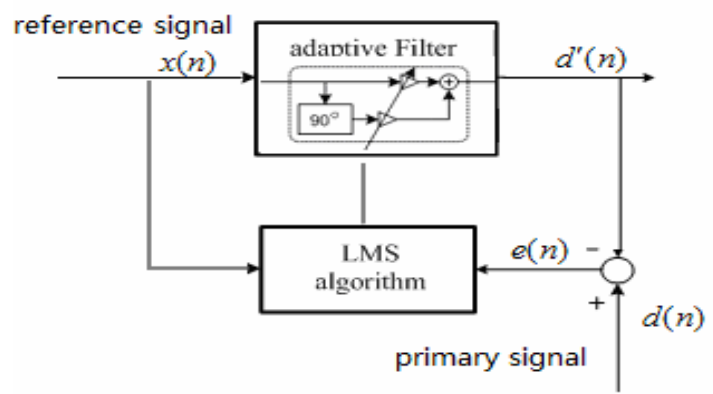

Fig.1 General form of adaptive notch filter noise canceller

For a sinusoidal reference signal $x_{n}=A \sin \left(2 \pi f_{r} \cdot n T+\phi\right)$ with frequency $f_{\mathrm{r}}$, sample time $T$, amplitude $A$ and phase shift $\phi$, the LMS algorithm updated equation is:

$$
\begin{gathered}
d_{n}=W_{n}^{\prime} \cdot x_{n} \\
\varepsilon_{n}=d_{n}-d_{n}^{\prime} \\
W_{n+1}=W_{n}+\alpha \cdot \varepsilon_{n} \cdot x_{n}
\end{gathered}
$$

Taking the z-transform of $d_{\mathrm{n}}$ and $\varepsilon_{\mathrm{n}}$, we can get the following equation:

$$
H(z)=\frac{E(z)}{D(z)}=\frac{z^{2}-2 z \cos \left(2 \pi f_{r} T\right)+1}{z^{2}-2\left(1-\alpha A^{2} / 2\right) z \cos \left(2 \pi f_{r} T\right)+1-\alpha A^{2}}
$$

Noting the Eq.4 , the zeros, $\mathrm{z}=\mathrm{e}^{ \pm \mathrm{j} 2 \pi f r T}$, are located on the unit circle while the poles are located on the same radial line and constrained by the coefficient $\alpha$ to be within the unit circle. The magnitude frequency characteristic for $D(\mathrm{z})$ to $E(\mathrm{z})$ has a deep valley at the frequency $f \mathrm{r}$, which result in the optimized attenuation of the component with frequency $f \mathrm{r}$ in the primary signal $d_{\mathrm{n}}$.

\section{Decoupling Control based on Modified Adaptive Notch Filter}

Inspired by the above adaptive notch filter, the decoupling control between DOFs is proposed, especially for the sinusoidal command waveform. The dynamics coupling between the translational and rotary DOFs can be shown in Fig.2. Here, the transfer function G21' and G12' represent the coupling from the translational DOF to rotary DOF and vise versa. 


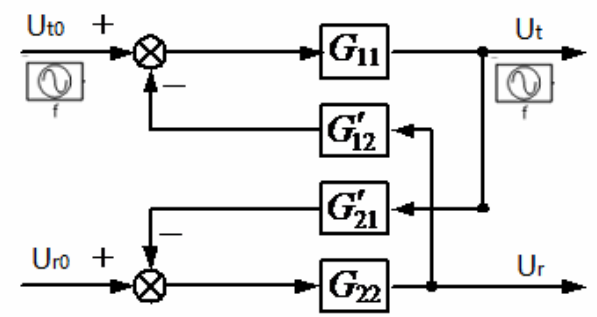

Fig. 2 Coupling relationship between translational and rotary DOF

For example, supposing the translational DOF is the primary signal, the sinusoidal waveform motion on the translational DOF will be coupled by the sinusoidal motion on the rotary DOF with the same frequency. Considering the coupling sinusoidal motion as the noise on the coupled DOF, the adaptive notch filer can be used to eliminate it. For our application, a modified adaptive notch filter is proposed, which is shown in Fig.3. Here the decoupling disturbance on the rotary DOF from the translation motion is eliminated to make sure the target translation motion of the shaking table.

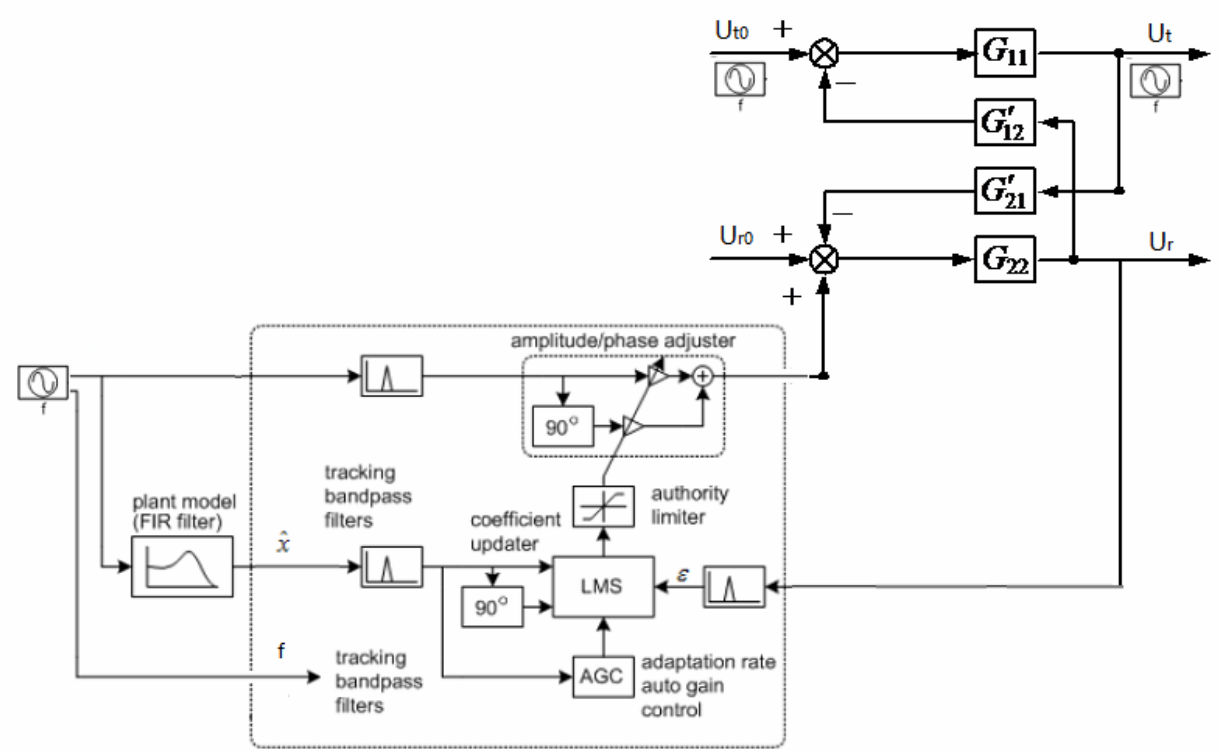

Fig.3 Modified adaptive notch filter applied to decoupling disturbance to rotary from translation

The amplitude/phase adjusting network for each frequency component is based on the trigonometric identity which states that a sinusoid of any amplitude $C$ and any phase can be obtained by the suitable choice of coefficients $A$ and $B$.

$$
A \sin (2 \pi f t)+B \cos (2 \pi f t)=C \sin (2 \pi f t+\phi)
$$

In the modified adaptive notch filter, these coefficients are adjusted using the Least Mean Square (LMS) algorithm to drive the response error to zero, here the normalized LMS algorithm is adopted.

Note that in the diagram a model of the plant is introduced to give the LMS algorithm the critical phase information needed to adjust canceller coefficients. The transfer function of this plant model must be measured online though a training procedure prior to cancelling harmonics disturbance noise.

Besides the sinusoidal waveform generator output and the plant response are filtered by narrowband band pass filters, which extract content from the signal only at the harmonic frequency. The center frequencies of these bandpass filters vary with the frequency of current command generator, allowing the modified adaptive notch filter work with sine sweep command waveforms. This frequency is also used to update the 90 degree phase shifters.

So the cross-coupling disturbance cancellling procedure can be summarized as :

1) Using the limited bandwidth random signal with small amplitude to train the plant FIR model from the primary DOF to the secondary DOF, which is benefit to the sweep sine command waveform. 
2) Start the primary function generator, and the secondary function generator will start as well because it is linked to the primary function generator.

3 ) Observe the secondary DOF's response, there will probably be a sizeable disturbance component at the fundamental frequency, i.e., the sine frequency of the primary function generator. Then enable the amplitude/phase adjusting network, and cancel this harmonic disturbance.

\section{Experimental results}

As the proof of concepts in previous sections, the decoupling control algorithm is implemented based on the rapid control prototyping technology and an experimental study is carried out on the redundant MAST. The test system consists of, as shown in Fig.4, 1 platform, 12 servo actuators, a hydraulic power supply and a computer system, etc.

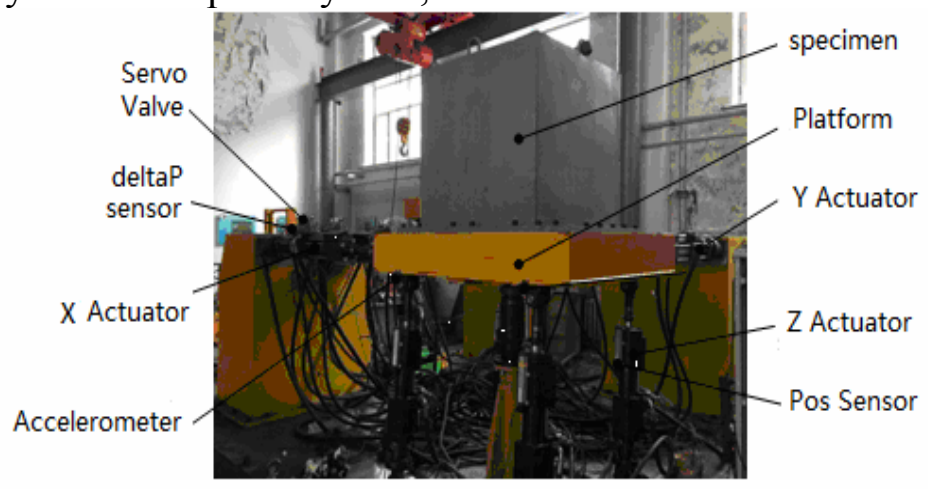

Fig.4 Redundant multi-axis shaking table

The primary signals in the experimental study are listed in Table 1 . The experimental results are demonstrated in Fig. 5 to Fig. 6.

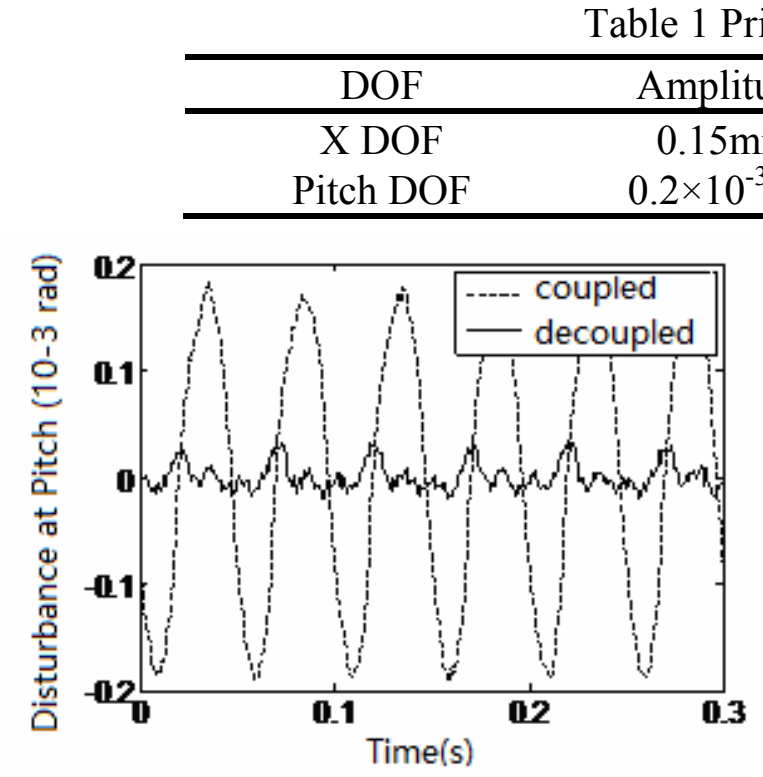

Table 1 Primary signals context 


\section{Summary}

Due to the overturning moment and off-center load of the MAST, the dynamics cross-coupling disturbance occurs when the motion on one translational DOF couples into another rotary DOF or vice versa. The proposed decoupling control, which is optimized to work with the sinusoidal command waveform, modifies the basic notch filter by providing the phase, and realizes the removal of the overturning moment or off-center load resonances. The experimental results show that the method can greatly reduce the coupled disturbance, and provides the improved control accuracy. Moreover, the decoupling control also can work with the sweep sine command waveform by using tracking band pass filter.

\section{References}

[1] R. T. Severn. The development of shaking tables - A historical note. Earthquake Engineering and Structural Dynamics. 40 (2011) 195-213.

[2] Zhou Hui-meng, Wu Bin. Validation study of shaking table hybrid test platform. Journal of Vibration Engineering. 26 (2013) 500-505.

[3] Wei wei, Yang zhidong, Gao Ting. Approximate Model Linearization Control for Hydraulic Hyper-redundant Shaking Table. 2013 International Conference on Mechatronic Sciences, Electric Engineering and Computer (MEC) Dec 20-22, 2013, Shenyang, China.

[4] Yang Zhidong, Li Hongren, Han Junwei. Linear Model and Inertia Decoupling of 3-Dimention Hydraulic shaking Table. Machine Tool and Hydraulic. 9(2005) 69-71.

[5] Wei wei, Yang zhidong, Han Junwei. Decoupling Control of Hyper-Redundant Shaking Table Based on Dynamic Coupling Model. Journal of South China University of Technology(Natural Science Edition). 42(2014) 124-130.

[6] Wei wei, Yang zhidong, Han Junwei. Modal space decoupling control for a hyper-redundant hydraulic shaking table. Jounal of harbin institute of technology (Natural Science Edition). 47(2015) 48-53.

[7] Tagawa Y, Kajiwara K. Controller development for the E-Defense shaking table. Journal of System and Control Engineering. 221(2007)171-181.

[8] D.B.Rao, S.Y.Kung. Adaptive notch filtering for the retrieval of sinusoids in noise. IEEE Transactions on Acoustics, Speech, and Signal Processing. 32(1984) 791-802.

[9] K. Hirano, S. Nishimura, S. Mitra. Design of Digital Notch Filters. IEEE Transactions on Communications. 22(1974) 964-970.

[10] E.H..W.Chan, R. A.Minasian. Photonic notch filter without optical coherence limitations. Journal of Lightwave Technology. 22(2004) 1811-1817.

[11] C.C.Tseng, S.C.Pei. Stable IIR notch filter design with optimal pole placement. IEEE. Transactions on Signal Processing. 49(2001) 2673-2681. 